\title{
A Tool for the Quantitative Anthropology of Music: Use of the nPVI Equation to Analyze Rhythmic Variability within Long-term Historical Patterns in Music
}

\author{
JOSEPH R. DANIELE[1] \\ University of California, Berkeley
}

\begin{abstract}
The development of musical style across time and geography is of particular interest to historians and musicologists, yet quantitative evidence to support these trends has been lacking. This paper illustrates a novel application of the nPVI ('normalized pairwise variability index') equation to probe and quantify the rhythmic components of music over time. The nPVI equation quantifies the average difference between adjacent events in a sequence (e.g. musical notes in a melody, successive vowels in a spoken sentence). Building upon an earlier finding that German/Austrian composer nPVI values increased steadily from 1600 to 1950 (while Italian composers showed no such increase), the nPVI 'distribution' of themes from individual composers was quantitatively explored. Interestingly, the proportion of 'low nPVI' or 'Italianate' themes decreases rapidly with time while 'high nPVI' (more Germanic) themes concomitantly increase in frequency. 'Middle range nPVIs' exhibit a constant incidence, arguing for a replacement of 'low nPVIs' (Italianate) with 'high nPVIs' over a short time instead of a more modest, long-term progressive shift. Thus, the precise rhythmic components of complex stylistic shifts in music can be quantitatively extracted from music and support the historical record and theory.
\end{abstract}

Submitted 2015 July 6; accepted 2015 November 4.

KEYWORDS: music rhythm, quantitative musicology, $n P V I$, historical trends, empirical

\section{INTRODUCTION}

THE nPVI ('normalized pairwise variability index') is an equation originally developed for linguistic analysis that quantifies the average amount of durational contrast between successive events in a sequence (e.g. musical notes in a melody or successive vowels in a spoken sentence). A high nPVI value, for instance, indicates greater durational contrast between adjacent events in a sequence (cf. the Appendix from Daniele \& Patel, 2013 for an example nPVI computation). Originally developed by phoneticians to show that "stress-timed" languages (British English, German, Dutch) had naturally higher nPVI values than "syllable-timed" languages (French, Spanish, Italian), Patel and Daniele (2003a) used the nPVI to show that a composer's native language directly influences the rhythms he/she writes (Grabe \& Low, 2002; Low, Grabe, \& Nolan, 2000; Ramus, 2002; for recent data on vocalic nPVI in English, German, Italian, and Spanish, see Arvaniti 2012, Figure 2b). Recent studies of the nPVI have begun to explore the variation of this statistic with respect to time and culture. More specifically, the work of Daniele and Patel has demonstrated that the nPVI can reveal patterns in the historical analysis of music (Daniele \& Patel, 2015; Daniele \& Patel, 2013; Daniele \& Patel, 2004; Patel \& Daniele, 2003b). By plotting mean nPVI vs. midpoint year for German/Austrian and Italian composers who lived between $\sim 1600$ and 1950 a steady increase in nPVI values was observed over this period for German/Austrian music while Italian music showed no such increase (Daniele \& Patel, 2013). These data proved to be consistent with the idea (from historical musicology) that the influence of Italian music on German music began to wane in the second half of the 1700s due to a rise of musical nationalism in Germany (Morrow, 1997). Of note, these findings have been replicated and expanded upon to include polynomial modeling of an initial increase and decrease in music from 34 French composers during this era (Hansen et al., 2015).

It is important to note that analyses such as those in Daniele and Patel (2004), and Daniele and Patel (2013) are based on assigning each composer a single, average nPVI value. More recently, when composer's lives were demarcated into different compositional epochs (by historical musicologists) Daniele and Patel (2015) found that the mean nPVI (for each compositional period) does not vary dramatically. Nevertheless, there is much still to learn by examining each composer's theme corpus more 
closely. For instance, what is the rhythmic "distribution" of each composer during this transition from 1760-1800? To what extent might historical factors (the "Italian" influence) affect nPVI distribution over time?

To test if nPVI could be used to detect dramatic historical shifts in music, the change in nPVI distribution was explored with respect to the aforementioned 1760-1800 stylistic transition in German music and compared to a culture where no change in nPVI would be expected (e.g. Italian). A simulation in which nPVI was modeled to increase modestly over a long period of time was then compared to the calculated data. Thus, this paper illustrates the utility of the nPVI equation and method in quantifying the precise rhythmic underpinnings of a complex stylistic transition in music history.

\section{METHODS}

\section{Theme List, Exclusion Criteria, and Data Analysis}

Data collection and analysis was computed and analyzed according to the previous methods and exclusion criteria were enumerated extensively in Daniele and Patel (2013). As in the Daniele and Patel studies, the musical materials for the current work were drawn from A Dictionary of Musical Themes, Revised Edition (Barlow \& Morgenstern, 1983). The midpoint year (the mathematical average of the birth and death year, representing when the composer was active) was considered in grouping these individuals into periods from the "Baroque/Classical Era" (1600-1750/1750-1810) which took place before and during the 17601800 transition to more stylistically "German" music and the "Romantic Era" (1825-1900) which followed this transition (Kmetz, Finscher, Schubert, Schepping, \& Bohlman, 2001). Beethoven was considered one of the Romantic composers despite writing before the "Romantic period" (Solomon, 1998; d'Indy, 1970). Modeling of nPVI distributions over time was completed using the "Normal Distribution Probability Calculator" in Sigma XL. More explicitly, distributions were calculated by plugging in composer mean $\mathrm{nPVI}$, standard deviation, and nPVI range into the aforementioned program and then plotting the resulting proportions for each range.

\section{RESULTS}

With the intent to chronicle the rhythmic underpinnings of German musical evolution the median nPVI of German/Austrian composers from different eras of musical history ("Baroque/Classical" 1600-1825, and "Romantic" 1825-1900) (Kmetz, Finscher, Schubert, Schepping, \& Bohlman, 2001; and see Methods) was calculated and used to categorize composers. Since these grouped data sets ("Baroque/Classical" composers (Bach, Haydn, Mozart)) and "Romantic" composers (Beethoven through R. Strauss, see Table 1) were deemed "non-normal" using the Anderson Darling Test for Normality, the medians (and not means) were used (nPVI $=32.2$ for the "Baroque/Classical" period and nPVI $=43.0$ for "Romantic"). These values were used to demarcate bins for each composer's theme corpus (Table 1). It is of note to mention that the nPVI at the intercept of the German/Austrian and Italian slopes (Daniele \& Patel, 2013) was 41.3 (for a 15 theme cutoff for Italians). Thus, the use of an nPVI of 43.0 as a demarcation point from the "Italian" style to the "German" style is reasonable.

Table 1. Percent of themes within a given nPVI tier for German/Austrian composers

\begin{tabular}{|c|c|c|c|c|c|c|c|}
\hline Composer & $\begin{array}{c}\# \\
\text { themes }\end{array}$ & $\begin{array}{l}\text { Mean } \\
\text { nPVI }\end{array}$ & $\begin{array}{l}\text { Midpoint } \\
\text { Year }\end{array}$ & $\begin{array}{l}\text { \% themes w/ } \\
\text { nPVI < } 32.2\end{array}$ & $\begin{array}{c}\% \text { themes w/ } \\
32.2<\text { nPVI }<43\end{array}$ & $\begin{array}{c}\% \text { themes } w / \\
\text { nPVI }>43\end{array}$ & $\begin{array}{l}\text { Natio- } \\
\text { nality }\end{array}$ \\
\hline J.S. Bach & 351 & 27.5 & 1717.5 & 65.8 & 16.2 & 17.9 & GER \\
\hline Haydn & 278 & 35.8 & 1770.5 & 48.9 & 21.2 & 29.9 & $\overline{\text { AUT }}$ \\
\hline Mozart & 460 & 41.7 & 1773.5 & 39.1 & 17.8 & 43.0 & AUT \\
\hline Beethoven & 487 & 43.0 & 1798.5 & 40.5 & 15.4 & 44.1 & $\overline{\text { GER }}$ \\
\hline Schubert & 232 & 47.1 & 1812.5 & 28.0 & 22.8 & 49.1 & $\overline{\text { AUT }}$ \\
\hline Mendelssohn & 145 & 39.9 & 1828 & 43.4 & 13.1 & 43.4 & GER \\
\hline Schumann & 216 & 41.2 & 1833 & 42.1 & 13.9 & 44.0 & GER \\
\hline Wagner & 82 & 63.9 & 1848 & 11.0 & 11.0 & 78.0 & GER \\
\hline
\end{tabular}




\begin{tabular}{|c|c|c|c|c|c|c|c|}
\hline $\begin{array}{c}\text { J. Strauss, } \\
\text { Jr. }\end{array}$ & 95 & 55.1 & 1862 & 18.9 & 14.7 & 66.3 & AUT \\
\hline Brahms & 362 & 43.5 & 1865 & 34.5 & 18.8 & 46.7 & GER \\
\hline R. Strauss & 111 & 60.0 & 1906.5 & 15.3 & 10.8 & 73.9 & GER \\
\hline
\end{tabular}

When the frequencies in Table 1 were plotted against each composer's midpoint year, and regression lines fitted, a striking trend was observed (Figure 1). The proportion of 'low nPVIs' $(<32.2)$ significantly decreased with time (Frequency $(\%)=-0.25^{*}$ (Midpoint Year) $+492.26, R^{2}=0.68, p<.01$ ) while the proportion of themes with higher nPVIs $(>43)$ significantly increased with time (Frequency $(\%)=$ $0.29 *$ (Midpoint Year) $-471.74, R^{2}=0.71, p<.01$ ). No significant change in the proportion of themes with nPVI 32.1 to 43.0 was seen ( $p=.14)$ suggesting that the majority of composers wrote the same percent of themes with nPVIs in this range. These trends are almost identical when the theme cutoff is raised to 100 themes, which excludes Wagner and Strauss, Jr. (data not shown). Thus, very low nPVI themes are seemingly being replaced with very high nPVI themes while the middle range stays constant. One does not see these same trends when this analysis is performed on individual Italian composers (a culture that has been shown to maintain a constant nPVI over time). For Italians, all slopes in these ranges are below (or equal to) 0.03 and none of the regressions are significant (data not shown).

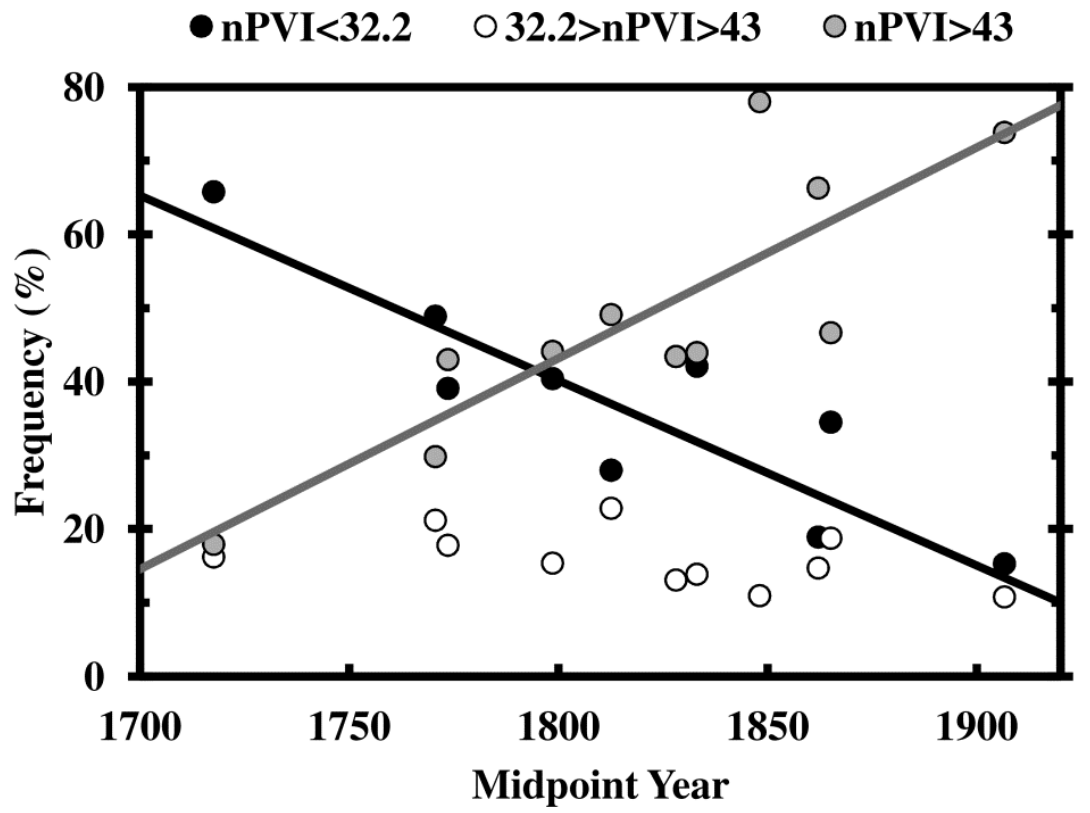

Fig. 1. Percent of themes within a particular range for each composer plotted against midpoint year (see Table 1). Three vertical dots (black, grey, and white), representing the percent of themes within a particular nPVI range for each composer are plotted against midpoint year. Linear regressions were computed from the proportion of themes, for each composer, within each nPVI range. Lines represent significant linear regressions $(p<.01)$. Minimum number of themes per composer $=75$.

While the Italian data gave important historical evidence for the potential uniqueness of this rhythmic shift in German/Austrian music, it was important to test what these nPVI proportions might look like if all composer nPVIs increased incrementally with time. A computer simulation was performed to test this hypothesis and the results are shown in Figure 2. Similar to the calculated composer data (Figure 1) the proportion of 'low nPVI' themes (>32.1) decreases in a significant manner (Frequency $(\%)=$ $0.22 *$ (Midpoint Yr) $+432.55, R^{2}=0.71, p<.01$ ) though the rate at is noticeably slower than the observed data (compare to slope 0.25, black line from Figure 1). The proportion of "high nPVI" themes (>43) also increases steadily and significantly (Frequency $(\%)=0.26^{*}$ (Midpoint Yr) $-423.53, R^{2}=0.70, p<.01$ ) yet this is also at a slower rate than the observed data (compare to slope 0.29, grey line from Figure 1). Finally, in stark contrast to the observed composer data (Figure 1) the proportion of "mid-range nPVI" themes $(32.2$ $>$ nPVI $>43)$ in the model decreases significantly with time (Frequency $(\%)=-0.04 *($ Midpoint $\mathrm{Yr})+$ $90.98, R^{2}=0.49, p=.017$ ) while the observed composer data showed no significant change. Almost identical results were found when Wagner and Strauss, Jr. were excluded. 


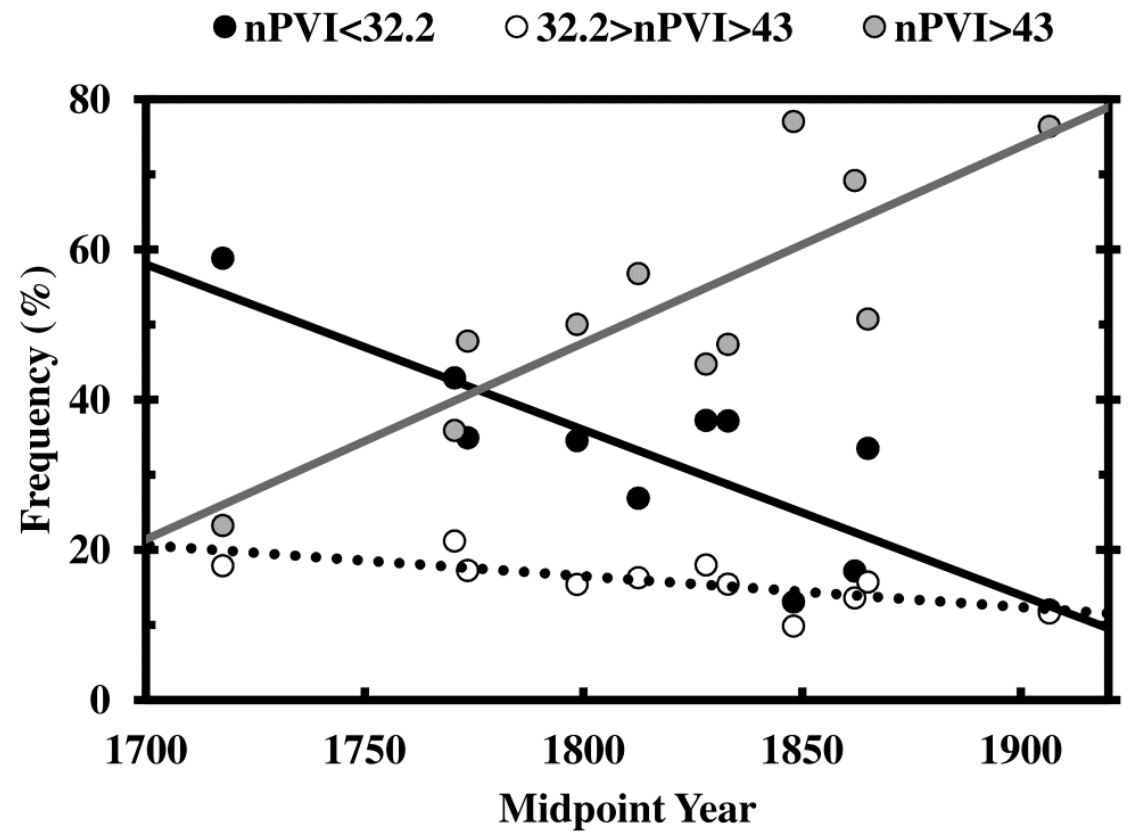

Fig. 2. Predicted rhythmic changes over time using simulation software. Predicted percent of themes within a particular range for each composer (based on each composer's calculated mean and standard deviation) plotted against midpoint year. Three vertical dots (black, grey, and white), represent the percent of themes within a particular nPVI range for each composer and are plotted against midpoint year. Linear regressions were computed from the proportion of themes, for each composer, within an nPVI range. In contrast to Figure 1, all regression lines are significant; dotted line $p=.017$, while solid lines are very significant $(p \leq .01)$.

\section{DISCUSSION}

The present study illustrates how nPVI analysis can reveal the precise rhythmic underpinnings of important musical shifts. Thus, to understand which rhythms are changing (and when) the distribution of nPVI within each composer's corpus of themes was explored.

\section{Evidence for "the Replacement Hypothesis"}

The suggestive increase in the mean nPVI of German/Austrian composers during the aforementioned 17601800 transition away from the Italian influence (Daniele \& Patel, 2013) prompted the development of a different method to study the "metric" of a composer's style, namely the distribution and spread of nPVI for all his/her themes (Table 1 and Figure 1). Testing the robustness and composition of this trend led to the observation that the frequency of themes with nPVI $<32.2$ drops dramatically with time. These themes were replaced with a concomitant increase in themes with nPVI $>43$. Themes in the middle range between 32.2 and 43 did not show any significant increase over time even when the theme cutoff was raised (Figure 1). A similar analysis of Italian composers (where one would expect no changes with time) revealed no significant linear regressions while a simulation of incremental nPVI increases with time also differed significantly from the observed results (Figure 2). These data offer suggestive evidence for the idea that 'low nPVI' themes in German music are being replaced with 'high nPVI' themes while the middle nPVI range stays constant ("the Replacement Hypothesis"). This is in contrast to an alternative hypothesis that the majority of nPVIs are modestly increasing to eventually resemble "more German" nPVIs by the mid- to late 1800 s (e.g. Figure 2). More broadly, these data suggest what has been reported by musicologists (e.g. Morrow, 1997) that composers in the late 1700's made a conscious choice to write "German" themes in place of the "Italianate" themes they once wrote.

\section{Suggestive Evidence for Incipient 20th Century Rhythms}


While most of the German/Austrian composers had a proportion of 'high nPVI' themes that ranged from $40-50 \%$ of themes, two composer's, Richard Wagner and Richard Strauss, had nearly $80 \%$ of their themes in this category. With such strikingly different distributions, it is interesting to speculate that these composers' themes might be reflecting rhythms that are characteristic of $20^{\text {th }}$ century composers, rather than the Romantic period they lived in. In fact, their style was most likely characteristic of the "New German School"; composers which pitted Beethoven's "absolute music" against their invention, the "symphonic poem". Importantly, these composers were not constrained by conventional musical forms e.g. sonata, concerto, etc. (Walker, 1993, pp. 338-367). This break from the traditional compositional style can be seen as early as Wagner's Tristan and Isolde which is thought to have inspired future musical conventions like atonality, often used in " $20^{\text {th }}$ century" music (Deathridge, 2008, p. 114). Future studies including additional "New German School" composers like Franz Liszt and $20^{\text {th }}$ century German composers like Arnold Schoenberg could test if this predilection for very high nPVI themes is prevalent in these artists.

In conclusion, the current paper demonstrates the significance of using nPVI analysis as a tool to probe and quantify the precise rhythmic components of stylistic trends in music history. Thus, it provides a quantitative mechanism for comparison and support to the historical record and theory. Additional research using nPVI analysis could include the exploration of not only how complex stylistic trends in music history progress and develop, but also what the specific, underlying rhythmic structure of these trends might be.

\section{NOTES}

[1] Correspondence - Department of Molecular and Cellular Biology, University of California, Berkeley, 188 Li Ka Shing Center, Rm 430E, Berkeley, CA 94530, email: jdaniele@berkeley.edu

\section{REFERENCES}

Arvaniti, A. (2012). The usefulness of metrics in the quantification of speech rhythm. Journal of Phonetics, 40, 351-373. https://doi.org/10.1016/j.wocn.2012.02.003

Barlow, H. \& Morgenstern, S. (1983). A dictionary of musical themes, revised edition. London: Faber and Faber.

Daniele, J.R. \& Patel, A.D. (2004). The interplay of linguistic and historical influences on musical rhythm in different cultures. In: S.D. Lipscomb et al. (Eds.), Proceedings of the 8th International Conference on Music Perception and Cognition, Evanston, IL, 2004 (pp. 759-762). Adelaide, Australia: Causal Productions.

Daniele, J. R., \& Patel, A. D. (2013). An empirical study of historical patterns in musical rhythm. Music Perception: An Interdisciplinary Journal, 31(1), 10-18. https://doi.org/10.1525/mp.2013.31.1.10

Daniele J.R. \& Patel, A.D. (in press). Stability and change in rhythmic patterning across a composer's lifetime: a study of four famous composers using the nPVI equation.

Music Perception.

Deathridge, J. (2008). Wagner Beyond Good and Evil. USA: University of California Press. https://doi.org/10.1525/california/9780520254534.001.0001

Grabe, E. \& Low, E.L. (2002) Durational variability in speech and the rhythm class hypothesis. In C. Gussenhoven \& N. Warner (Eds.), Laboratory Phonology 7 (pp. 515-546). Berlin: Mouton de Gruyter. https://doi.org/10.1515/9783110197105.515

Hansen, N.C., Sadakata, M., \& Pearce, M. (In Press). Non-linear changes in the rhythm of European art music: Quantitative support for historical musicology. Music Perception

Huron, D. \& Ollen, J. (2003). Agogic contrast in French and English themes: Further support for Patel and Daniele. Music Perception, 21, 267-271. https://doi.org/10.1525/mp.2003.21.2.267 
Kmetz, J., Finscher, L., Schubert, G., Schepping, W., \& Bohlman, P. V. (2001). Germany. In S. Sadie (Ed), The New Grove dictionary of Music and Musicians (Vol. 9, pp. 708-744). New York: Grove.

London, J. \& Jones, K. (2011) Rhythmic refinement to the nPVI measure: a reanalysis of Patel \& Daniele (2003a). Music Perception, 29, 115-120. https://doi.org/10.1525/mp.2011.29.1.115

Low, E.L., Grabe, E. \& Nolan, F. (2000). Quantitative characterisations of speech rhythm: Syllable-timing in Singapore English. Language and Speech, 43(4), 377-401.

https://doi.org/10.1177/00238309000430040301

McGowan R.W. \& Levitt A.G. (2011) A comparison of rhythm in English dialects and music. Music

Perception, 28, 307-311. https://doi.org/10.1525/mp.2011.28.3.307

Morrow, M. S. (1997). German music criticism in the late eighteenth century: aesthetic issues in instrumental music. Cambridge, U.K: Cambridge University Press. https://doi.org/10.1017/CBO9780511549427

Patel, A.D. \& Daniele, J.R. (2003a). An empirical comparison of rhythm in language and music. Cognition, 87, B35-B45. https://doi.org/10.1016/S0010-0277(02)00187-7

Patel, A.D. \& Daniele, J.R. (2003b). Stress-timed vs. syllable-timed music? A comment on Huron and Ollen (2003). Music Perception, 21, 273-276. https://doi.org/10.1525/mp.2003.21.2.273

Ramus, F. (2002). Acoustic correlates of linguistic rhythm: Perspectives. In: B. Bell \& I. Marlien (Eds.) Proceedings of Speech Prosody, Aix-en-Provence (pp. 115-120). Aix-en-Provence: Laboratoire Parole et Langage.

Ramus, F., Nespor, M. \& Mehler, J. (1999). Correlates of linguistic rhythm in the speech signal. Cognition, 73, 265-292. https://doi.org/10.1016/S0010-0277(99)00058-X

Walker, A. (1987). Franz Liszt: The Weimar Years, 1848-1861. Cornell University Press. 